\title{
Vector Bundles on an Infinitesimal Neighborhood of a Negative and Split Plane
}

\author{
E. Ballico ${ }^{1}$ \\ Dept. of Mathematics \\ University of Trento \\ 38050 Povo (TN), Italy \\ ballico@science.unitn.it
}

\begin{abstract}
Let $W$ be an $n$-dimensional complex manifold or a smooth algebraic variety and $Z \subset W$. Assume $Z \cong \mathbf{P}^{2}, n \geq 3$ and that the normal bundle $N_{Z, W}$ of $Z$ in $W$ is a direct sum of line bundles with negative degree. Let $Z^{(\infty)}$ denote the formal completion of $W$ along $Z$. Here we compute the dimension of the deformation space of several "general " vector bundles $A$ on $Z^{(\infty)}$ in terms of the splitting type of $N_{Z, W}$ and the integers $c_{1}(A \mid Z)$ and $c_{2}(A \mid Z)$.
\end{abstract}

\section{Mathematics Subject Classification: 14J60}

Keywords: vector bundle on $\mathbf{P}^{2}$, infinitesimal neighborhood, normal bundle

\section{VeCtor BUNDLES ON AN INFINITESIMAL NEIGHBORHOOD OF A NEGATIVE AND SPLIT PLANE}

Let $W$ be either an $n$-dimensional complex manifold or a smooth $n$-dimensional algebraic variety. Assume $n \geq 3$ and the existence of $Z \subset W$ such that $Z \cong \mathbf{P}^{2}$ (in the holomorphic or the algebraic category) such that the nornal bundle $N_{Z, W}$ of $Z$ in $W$ is isomorphic to the direct sum of $n-2$ line bundles with negative degree. Let $Z^{\infty}$ denote the formal completion of $W$ along $Z$. Here we compute the dimension of the deformation space of several "general " vector bundles $A$ on $Z^{(\infty)}$ in terms of the splitting type of $N_{Z, W}$ and the integers $c_{1}(A \mid Z)$ and $c_{2}(A \mid Z)$. We will first describe that quite often this deformation space is smooth.

Remark 1. Fix an integer $m \geq 0$, a vector bundle $E_{m}$ on $Z^{(m)}$ and a vector bundle $F$ on $Z$. Set $E:=E_{m} \mid X$ and assume that $E$ is simple, i.e. assume $h^{0}(Z, \operatorname{End}(E))=1$. Since $N_{Z, W}{ }^{*}$ is a direct sum of line bundles with negative degree and $\omega_{Z}$ has degree -4 , Serre duality implies $h^{2}(Z, \operatorname{End}(E) \otimes$

\footnotetext{
${ }^{1}$ The author was partially supported by MIUR and GNSAGA of INdAM (Italy).
} 
$\left.S^{x}\left(N_{Z, W^{*}}\right)\right)=0$ for all $x \geq 0$. This vanishing for $x=m$ implies the existence of a vector bundle $E_{m+1}$ on $Z^{(m+1)}$ such that $E_{m+1} \mid X^{(m)} \cong E_{m}$ ([3], Sazt 1$)$. Since $N_{Z, W}{ }^{*}$ is ample, $h^{1}\left(X, \operatorname{End}(F) \otimes S^{t}\left(N_{Z, W}{ }^{*}\right)\right)=0$ for $t \gg 0$. and hence $\gamma(E):=\sum_{m \geq 0} h^{1}\left(X, \operatorname{End}(F) \otimes S^{m}\left(N_{Z, W}{ }^{*}\right)\right)<+\infty$. There exists a vector bundle $G$ on $Z^{(\infty)}$ such that $G \mid Z \cong F$ and for a fixed such $G$ the deformation space of $G$ is an open neighborood of $0 \in \mathbb{C}^{\gamma(E)}$ ([4], Satz 2 and first Bemerkung at p. 115). There is a vector bundle $A$ in an analytic neighborhood $U$ of $X$ in $W$ such that $A \mid Z^{(\infty)}=G$ and hence $A \mid Z \cong F$ ([4], Satz 3). Now fix any vector bundle $A$ on $Z^{(\infty)}$ and set $E:=A \mid Z$ and $\beta(E)=\gamma(E)$. Thus

$$
\beta(E)=\gamma(A)=\sum_{m \geq 0} h^{1}\left(Z, E n d(E) \otimes S^{m}\left(N_{Z, W}{ }^{*}\right)\right) .
$$

Our aim will be the computation of the integer $\beta(E)$ when $A$ and $E$ have rank two and $E$ is simple (but not necessarly stable). Since $N_{Z, W}{ }^{*}$ is a direct sum of line bundles, to compute $\beta(E)$ (for any such fixed $N_{Z, W}$ ) it is sufficient to compute all integers $\beta(E, t):=h^{1}(Z, \operatorname{End}(E)(t)), t \in \mathbb{N}$.

Set $\mathcal{O}:=\mathcal{O}_{\mathbf{P}^{2}}$. For any sheaf $A$ on $\mathbf{P}^{2}$, set $h^{i}(A):=h^{i}\left(\mathbf{P}^{2}, A\right)$.

Example 1. Fix integers $s, c_{1}$ such that $s>0$ and $S \subset \mathbf{P}^{2}$ such that $\sharp(S)=$ $s$. If $c_{1} \geq 2$ assume $h^{0}\left(\mathcal{I}_{S}\left(c_{1}-1\right)\right)=0$. Notice that for all $c_{1}$ we have $h^{0}\left(\mathcal{I}_{S^{\prime}}\left(c_{1}-3\right)\right)=0$ for all $S^{\prime} \subset S$ such that $\sharp\left(S^{\prime}\right)=s-1$. Hence the socalled Cayley-Bacharach condition is satified and hence there is a rank 2 vector bundle on $\mathbf{P}^{2}$ fitting in the the case $t=0$ (and hence in the general case) of the following exact sequence $([1])$ :

$$
0 \rightarrow \mathcal{O}(t) \rightarrow E(t) \rightarrow \mathcal{I}_{S}\left(t+c_{1}\right) \rightarrow 0
$$

If $h^{0}\left(\mathcal{I}_{S}\left(c_{1}\right)\right)=0$, then $E$ is simple. In particular we get that $E$ is always simple if $c_{1} \leq 0$. If $c_{1}>0$, then we may take $E$ stable (and hence simple) if $S \subset \mathbf{P}^{2}$ is general and the extension (2) is general ([1]). We have $\operatorname{rank}(E)=2$, $c_{1}(E)=c_{1}$ and $c_{2}(E)=s$. Hence $E^{*} \cong E\left(-c_{1}\right)$. Thus $\operatorname{End}(E) \cong E\left(-c_{1}\right)$. Thus tensoring (2) with $E^{*}(t)$ we get the following exact sequence

$$
0 \rightarrow E\left(t-c_{1}\right) \rightarrow \operatorname{End}(E)(t) \rightarrow \mathcal{I}_{S} \otimes E(t) \rightarrow 0
$$

Since $\sharp(S)=s$ and $E$ has $\operatorname{rank} 2, h^{0}\left(\mathcal{I}_{S} \otimes E(t)\right) \geq \max \left\{0, h^{0}(E(t))-2 s\right\}$ for all $t \in \mathbb{Z}$. By Serre's vanishing theorem we a priori know that $h^{0}\left(\mathcal{I}_{S} \otimes\right.$ $E(t))=h^{0}(E(t))-2 s$ for $t \gg 0$. From $(2)$ we get $h^{0}(E(t)) \geq h^{0}\left(\mathcal{I}_{S} \otimes E(t)\right) \geq$ $(t+2)(t+1) / 2+h^{0}\left(\mathcal{I}_{S}\left(c_{1}+t\right)\right)$ for all integers $t>0$. Let $z$ be the first integer $\geq \max \left\{0,-c_{1}\right\}$ such that $s<(z+c-1+2)\left(z+c_{1}+1\right) / 2$. We always assume $h^{0}\left(\mathcal{I}_{S}\left(z+c_{1}-1\right)\right)=0$. This is true if $S$ is general. From (2) we get $h^{0}(E(t))=(t+2)(t+1) / 2$ for all $t<z$, while $h^{0}(E(w))>(w+2)(w+1) / 2$ for all $w \geq z$. Thus $h^{0}\left(\mathcal{I}_{S} \otimes E(t)\right)=(t+2)(t+1) / 2$ for all $0 \leq t<z$. For any $P \in \mathbf{P}^{2}$ let $2 P$ denote the first infinitesimal neighborhood of $P$ in $\mathbf{P}^{2}$, i.e. the closed subscheme of $\mathbf{P}^{2}$ with $\left(\mathcal{I}_{\{P\}}\right)^{2}$ as its ideal sheaf. For any finite $B \subset \mathbf{P}^{2}$ set $2 B:=\cup_{P \in B} 2 P$. Fix $a \in H^{0}\left(\mathcal{I}_{S} \otimes E(t)\right)$ such that $a$ is not in the image of 
$H^{0}(\mathcal{O}(t))$ by the map induced in cohomology by (2). Let $a^{\prime} \in H^{0}\left(\mathcal{I}_{S}\left(t+c_{1}\right)\right)$ be the image of $a$ by the map induced in cohomology by (2). By assumption we have $a^{\prime} \neq 0$.

Claim: $a^{\prime} \in H^{0}\left(\mathcal{I}_{2 S}\left(t+c_{1}\right)\right)$.

Proof of the Claim: It is sufficient to prove that $a^{\prime} \in H^{0}\left(\mathcal{I}_{2 P}\left(t+c_{1}\right)\right)$ for all $P \in S$. Fix $P \in S$. It is sufficient to show that $a^{\prime} \mid D$ vanishes of order at least 2 at $P$ for every germ at $P$ of a smooth curve $D$ passing through $P$. Call $b$ the image in $H^{0}(E(t))$ of the section of $E$ inducing the extension (2) for $t=0$ multiplied by a degree $t$ homogeneous form not vanishing at $P$. Let $\mathbf{m}$ denote the maximal ideal of the local ring $\mathcal{O}_{D, P}$. Chosing a local basis of $E$ at $P$ we may see the pair $(a, b)$ as a $2 \times 2$ matrix with entries in $\mathbf{m}$. Hence its determinant vanishes with order at least 2 at $P$, proving the Claim.

From now on we assume that $S$ is general in $\mathbf{P}^{2}$. This assumption implies $h^{0}\left(\mathcal{I}_{2 S}(x)\right)=\max \{0,(x+2)(x+1) / 2-3 s\}$ and $h^{1}\left(\mathcal{I}_{2 S}(x)\right)=\max \{0,3 s-$ $(x+2)(x+1) / 2\}$ for all integers $x \geq 3$, unless $(s, x)=(5,4)([2])$. We have $h^{0}\left(\mathcal{I}_{2 S}(4)\right)=h^{1}\left(\mathcal{I}_{2 S}(4)\right)=1$ if $s=6$. We have $h^{0}\left(\mathcal{I}_{2 S}(2)\right)=0$ if $s \geq 3$, $h^{0}\left(\mathcal{I}_{2 S}(2)\right)=1$ if $s=2$ and $h^{0}\left(\mathcal{I}_{2 S}(2)\right)=2$ if $s=1$. We have $h^{0}\left(\mathcal{I}_{2 S}(1)\right)=0$ for all $s>0$. Since $S$ is general, we get $h^{0}(E(t))=(t+2)(t+1) / 2$ for all $0 \leq t<z$ and $h^{0}(E(t))=(t+2)(t+1)-s$ for all $t \geq z$. Let $\eta$ be the first integer $\geq \max \left\{0,-c_{1}\right\}$ such that $(\eta+2)(\eta+1) \geq 6$ s. If $\left(s, \eta+c_{1}\right) \notin$ $\{(2,2),(5,4)\}$, then the Claim and [2] imply $h^{0}\left(\mathcal{I}_{S} \otimes E(t)\right)=(t+2)(t+1) / 2$ for all $0 \leq t<\eta$ and $h^{0}\left(\mathcal{I}_{S} \otimes E(t)\right)=(t+2)(t+1)-3 s$ for all $t \geq \eta$. Thus $h^{0}\left(\mathcal{I}_{S} \otimes E(t)\right)=h^{0}(E(t))-2 s$ for all $t \geq \eta$.

We summarize Example 1 in the following statements.

Theorem 1. Fix integers $s, c_{1}$ such that $s>0$. If $c_{1} \geq 0$, then assume $s>$ $c_{1}\left(c_{1}+1\right) / 2$. Let $\eta$ be the first integer $\geq \max \left\{0,-c_{1}\right\}$ such that $(\eta+2)(\eta+1) \geq$ 6s. Assume $\left(s, \eta+c_{1}\right) \notin\{(2,2),(5,4)\}$. Let $S \subset \mathbf{P}^{2}$ be a general subset with $\sharp(S)=s$ and $E$ any vector bundle fitting in the exact sequence (2). Set $\beta(E, t):=H^{0}\left(\mathcal{I}_{S} \otimes E(t)\right)$. Then $\beta(t)=(t+2)(t+1) / 2$ for all $0 \leq t<\eta$ and $\beta(E, t)=(t+2)(t+1) / 2-3 s=h^{0}(E(t))-2 s$ for all $t \geq \eta$.

Theorem 2. Let $W$ be an $n$-dimensional complex manifold, $n \geq 3$, and $Z \subset$ $W$ such that $Z \equiv \mathbf{P}^{2}$ and the conormal bundle of $Z$ in $W$ is the direct sum of $n-2$ line bundles of degree $a_{1} \geq \cdots \geq a_{n-2}>0$. Fix integers $s, c_{1}$ such that $s>0$. If $c_{1} \geq 0$, then assume $s>c_{1}\left(c_{1}+1\right) / 2$. Let $\eta$ be the first integer $\geq \max \left\{0,-c_{1}\right\}$ such that $(\eta+2)(\eta+1) \geq 6$ s. Assume $\left(s, \eta+c_{1}\right) \notin$ $\{(2,2),(5,4)\}$. Let $S \subset \mathbf{P}^{2}$ be a general subset with $\sharp(S)=s$ and $E$ any vector bundle fitting in the exact sequence (2). For any integer $m>0$ set $\Sigma(m, n-2):=\left\{\left(i_{1}, \ldots, i_{n-2}\right) \in \mathbb{N}^{n-2}: i_{1}+\cdots+i_{n-2}=m\right\}$. For every $\Sigma \in \Sigma(m, n-2)$ set $\Sigma\left(a_{1}, \ldots, a_{n-2}\right)=i_{1} a_{1}+\cdots+i_{n-2} a_{n-2}$. There is a vector bundle $A$ on $Z^{(\infty)}$ such that $A \mid Z \cong E$ and any such a bundle $A$ has a smooth 
deformation space of dimension

$$
\begin{gathered}
1+\sum_{m>0} \sum_{\Sigma \in \Sigma(m, n-2)} \beta\left(\Sigma\left(a_{1}, \ldots, a_{n-2}\right)\right) . \\
\text { REFERENCES }
\end{gathered}
$$

[1] J. Brun, Les fibrés de rang deux sur $\mathbf{P}^{2}$ et leurs sections, Bull. Soc. Math. France 108 (1980), no. 4, 457-473.

[2] K. A. Chandler, A brief proof of a maximal rank theorem for generic double points in projective space, Trans. Amer. Math. Soc. 354 (2001), no. 5, 1907-1920.

[3] Th. Peternell, Vectorraumbündel in der Nähe von kompakten complexen Unterräumen, Math. Ann. 257 (1981), no. 1, 111-134.

[4] Th. Peternell, Vectorraumbündel in der Nähe von exzeptionellen Unterräumen - das Modulproblem, J. Reine Angew. Math. 336 (1982), 110-123.

Received: July 18, 2006 